\title{
Indications of a New Antibiotic in Clinical Practice: Results of the Tigecycline Initial Use Registry
}

\author{
Daniel Curcio ${ }^{1}$, Francisco Fernández ${ }^{1}$, Alejandro Cané $^{2}$, Laura Barcelona ${ }^{3}$, Daniel Stamboulian ${ }^{3}$ \\ and the Tigecycline Initial Use Registry Group \\ ${ }^{1}$ San José Hospital, Infectious Diseases Department; ${ }^{2}$ Wyeth SA.; ${ }^{3}$ Center of Research in Infectious Diseases; Buenos Aires, Argentina
}

\begin{abstract}
Tigecycline is the first of a new class of antibiotics named glycylcyclines and it was approved for the treatment of complicated intra-abdominal infections and complicated skin and skin structure infections. Notwithstanding this, tigecycline's pharmacological and microbiological profile which includes multidrug-resistant pathogens encourages physicians' use of the drug in other infections. We analyzed, during the first months after its launch, the tigecycline prescriptions for 113 patients in 12 institutions. Twenty-five patients $(22 \%)$ received tigecycline for approved indications, and $88(78 \%)$ for “off label” indications $(56 \%$ with scientific support and $22 \%$ with limited or without any scientific support). The most frequent “off label” use was ventilator associated pneumonia (VAP) (63 patients). The etiology of infections was established in 105 patients (93\%). MDR-Acinetobacter spp. was the microorganism most frequently isolated (50\% of the cases). Overall, attending physicians reported clinical success in 86 of the 113 patients (76\%). Our study shows that the "off label” use of tigecycline is frequent, especially in VAP. due to MDRAcinetobacter spp., where the therapeutic options are limited (eg: colistin). Physicians must evaluate the benefits/ risks of using this antibiotic for indications that lack rigorous scientific support.

Key-Words: Tigecycline, off-label, ventilator-associated pneumonia.
\end{abstract}

In, 2006 the Information System of Bacterial Resistance (SIR), which includes 27 centers from Argentina, published worrisome rates of nosocomial bacterial resistance: $50 \%$ of methicillin-resistant Staphylococcus aureus (MRSA), 50\%$70 \%$ of Klebsiella pneumoniae resistant to third-generation cephalosporins, and 25\% of Pseudomonas aeruginosa and $60 \%$ of Acinetobacter spp. carbapenem-resistant [1].

This increasing medical issue calls for a more effective solution by means of new antimicrobial agents, especially those with novel mechanisms of action and activity against multidrug-resistant (MDR) bacteria.

Tigecycline is the first of a new class of antibiotics named glycylcyclines and is active in vitro against a variety of Grampositive and Gram-negative organisms, including nosocomial resistant pathogens such as MRSA, extended spectrum $\beta$ lactamases (ESBLs)-producing Enterobacteriaceae (EB), and MDR-Acinetobacter spp [2].

It has been approved by the US Food and Drug Administration (FDA) for the treatment of complicated intraabdominal infections (cIAI), and complicated skin and skin structure infections (cSSSI) [2]. Notwithstanding this, tigecycline's pharmacological and microbiological profiles encourage physicians' use of the drug in other infections caused by resistant pathogens featuring limited therapeutic options.

Considering this, we designed the Tigecycline Initial Use Registry (TIUR), a multicenter prospective observational study designed to characterize the indication types,

Received on 15 December 2007; revised 29 April 2008.

Address for correspondence: Dr. Daniel Curcio, M.D.Santo Tomé 5239 piso 4to 22 (1419) Ciudad Autónoma de Buenos Aires, Argentina. Phone: (+54 11) 4567-4426. Fax: (+54 11) 4822-2748. E-mail (1): djcurcio@gmail.com.E-mail (2): infectologia.institucional@gmail.com Financial support: This study was conducted with an independent unrestricted grant from Wyeth, Argentina.

The Brazilian Journal of Infectious Diseases 2008;12(3):198-201. (C) 2008 by The Brazilian Journal of Infectious Diseases and Contexto Publishing. All rights reserved. pathogens, and outcomes of patients who were treated with tigecycline in 12 Argentinean institutions.

We hypothesized that a systematic analysis of tigecycline prescriptions in our country might help us understand the clinical outcomes of patients who were treated for indications other than cIAI and cSSSI.

\section{Material and Methods}

Study Design

The TIUR included patients treated with tigecycline from November 1, 2006 to March 31, 2007. Patients were eligible for the TIUR if they received at least one dose of tigecycline and were not part of a clinical trial.

Attending physicians collected, in an ad hoc case report form, the following patient's information: sex, age, admission setting (general ward or intensive care unit), infection type for which tigecycline was indicated, severity of illness at admission (measured by the Mortality Probability Model MPM- II ${ }_{0}$ score [3]), microbiological documentation, previous and concomitant antibiotic therapy -defined as a patient who received at least one dose of another antibiotic before or during the treatment with tigecycline, respectively- and clinical outcome to the tigecycline treatment.

To analyze the data, we divided the tigecycline indications into three categories: type I, labeled indications (cIAI and cSSSI); type II, not approved indications ("off label”), with pharmacological and microbiological evidence that the drug is useful for a particular indication (eg. community-acquired pneumonia and hospital-acquired pneumonia); and type III, "off label" indications with, up to the moment, insufficient data regarding that particular use of the drug (eg. bacteremia, osteomyelitis).

\section{Study Population}

The analysis was restricted to patients whose form had been accurately completed by their attending physicians. 


\section{Outcome Assessment}

The attending physician evaluated the patient's clinical response to therapy as: cure if the patient's resolution of signs and symptoms was such that no further antibiotic therapy was required; improved if a patient showed partial resolution of clinical signs and symptoms; failure if the patient had an inadequate response to therapy; and undetermined if no evaluation was possible for any reason (e.g. a patient who received tigecycline for less than $72 \mathrm{hs}$ ). Clinical success (CS) was defined as an outcome of cure or improved.

\section{Statistics}

Results are expressed as proportions and presented with their corresponding 95\% confidence interval. When applicable, two tailed hypothesis testing for difference in proportions was used.

\section{Results}

Twelve institutions participating in the TIUR reported a total of 133 patients receiving at least one dose of tigecycline. The population under study consisted of 113 patients (84.9\%). Twenty patients were excluded from the analysis because the case report form was not complete.

Only 25 patients (22\%) received tigecycline for labeled indications (type I). Eighty- eight patients (78\%) received tigecycline for "off label” indications (type II 56\% -63/113and type III 22\% -25/113). The most frequent "off label” indication was ventilator-associated pneumonia (VAP) (100\% of type II indications) (Table 1).

Patients' mean age was 58 years (range 17-93) without significant differences between indications. Sixty-two patients were male (55\%) (Table 1).

Patients in the type-II indication group were admitted more frequently to intensive care unit (ICU) (98\%) than those included in type I or III indication groups $(\mathrm{p}<0.0001)$; showing MPM-II score values significantly higher than patients of type I and III indications (52 vs. 32 and 29 respectively, $\mathrm{p}=0.0001$ ). The length of stay for patients with type III indications was significantly higher than for those with type I and II indications (57 days vs. 35 and 38 days respectively, $\mathrm{p}=0.05$ ) (Table 1 ).

Of the 113 patients, 149 isolates were identified. MDRAcinetobacter spp. (only susceptible to colistin and minocycline) was the microorganism most frequently isolated (50\% of the cases) whose proportion rates were significantly higher in patients with type II indications (VAP) than in those with type I and III indications ( $\mathrm{p}=0.0005)$ (Table 1 ).

There was no significant difference between the three indication groups regarding the proportion of patients with previous and concomitant antibiotic therapy (Table 1). Vancomycin, carbapenems (imipenem or meropenem) and piperacillin-tazobactam, alone or in combination, were the most frequent antibiotics previously used. The most common concomitant antibiotics were antipseudomonal antibiotics (colistin, carbapenems -imipenem or meropenem-, ceftazidime, amikacin and ciprofloxacin).
The evaluated patients received tigecycline (initial dose of $100 \mathrm{mg}$ and then $50 \mathrm{mg}$ every $12 \mathrm{~h}$ ) for an average of 14 days (range 1-67). The median length of treatment for type III indications (21 days) was significantly higher than type I (15 days) and type II (11 days) indications ( $p=0.015$ ) (Table 1$)$.

Overall, attending physicians reported CS in 86 of the 113 patients (76\%, range 68-84\%). The CS rates observed in type I and III indications was significantly higher than in type II indications (88\% and 84\% vs. 66\% respectively, $\mathrm{p}=0.032$ ) (Table 2).

The clinical success rate showed no significant difference between the 82 patients with prior antibiotic therapy and 31 patients without such therapy (74\% in both cases). In contrast, the success rate was markedly different in 35 patients with concomitant antibiotic therapy and 78 without such treatment (57\% and 95\% respectively, $\mathrm{p}<0.00001$ ) (Table 2).

Global CS (76\%) was not affected by the presence of MDRAcinetobacter spp. (67\%, range 56-77\%), SAMR (68\%, range 47-85\%), or ESBLs-producing enterobacteria (81\%, range 62$100 \%)$. Patients with VAP (100\% of type II indications) due to MDR-Acinetobacter spp. showed a CS rate of 64\% (range 52$75 \%)$.

In patients with CS, the median of duration of tigecycline therapy and the lengths of stay were 16 days and 42 days, respectively. Taking into account the different indications, patients with type III indications had a duration of treatment with tigecycline significantly longer than type I and type II indications (22 days vs. 15 and 12 days, respectively, $\mathrm{p}=0.015$ ) (Table 2).

Global mortality proportion was 26.5\% (30/113 patients). The crude mortality of type II indications was significantly higher than type I and III indications (38\% -24/63- vs. 12\% -3/ 25 - and $12 \%-3 / 25$ - respectively, $\mathrm{p}=0.037$ ).

\section{Discussion}

Our study (TIUR) provides information about how tigecycline was used in clinical practice during the first months after its commercialization in Argentina.

This analysis showed that tigecycline was commonly used for "off label” indications (78\%), especially in patients admitted to ICU (78\%) with VAP (56\%) due to MDR-Acinetobacter spp. (61\%).

"Off label” indication use is defined as prescribing the drug for an indication other than the one approved by regulatory authorities. The American Medical Association estimates that $40 \%-60 \%$ of all prescriptions in the United States are issued for drugs being used in a fashion other than their approved purpose with “off-label” prescribing being particularly common for infectious diseases [4].

The mere $22 \%$ use of tigecycline for labeled indications, in our country, could be based on the other effective and less expensive antibiotic treatment options which physicians have for treating $\mathrm{CIAI}^{22}$ and $\mathrm{cSSSI}^{23}$. In contrast, ICU-physicians have the daily challenge of treating patients with VAP due to MDR-bacteria. 
Table 1. Patient demographic and clinical characteristics.

\begin{tabular}{|c|c|c|c|c|}
\hline \multirow[t]{2}{*}{ Characteristics } & \multirow[t]{2}{*}{ Global } & \multicolumn{3}{|c|}{ Indications } \\
\hline & & Type I & TypeII & TypeIII \\
\hline Number of patients, n (\%) & $113(100)$ & $\begin{array}{l}25(22) \\
- \text { cSSSI }^{1} 17 \\
- \text { cIAI }^{2} 8\end{array}$ & $\begin{array}{l}63(56) \\
- \text { VAP }^{3} 63\end{array}$ & $\begin{array}{l}25(22) \\
\text {-bacteremia } 12 \\
\text {-osteomyelitis } 4 \\
\text {-mediastinitis } 2 \\
\text {-others } 7\end{array}$ \\
\hline Age; mean years (range) & 58 (17-93) & 52 (19-93) & 62 (17-89) & 55 (29-79) \\
\hline Male; n (\%) & $62(55)$ & $13(52)^{4}$ & $37(59)^{5}$ & $14(54)^{6}$ \\
\hline ICU7 location, n (\%) & 88 (78) & $10(40)^{8}$ & $62(98)^{9}$ & $16(64)^{10}$ \\
\hline MPM $\mathrm{II}_{0}{ }^{11}$, median $(\dagger)^{12}$ & 45 (38) & $32(14)^{13}$ & $52(55)^{14}$ & $29(11)^{15}$ \\
\hline $\mathrm{LOS}^{16}$, median & 41 & $35^{17}$ & $38^{18}$ & $57^{19}$ \\
\hline Isolates, $\mathrm{n}$ & 149 & 37 & 82 & 30 \\
\hline$-A B-M^{20}{ }^{20}(\%)$ & $74(50)$ & $12(32)^{21}$ & $50(61)^{22}$ & $12(40)^{23}$ \\
\hline$-\mathrm{MRSA}^{24}(\%)$ & $25(17)$ & $9(24)$ & 9 (11) & 7 (23) \\
\hline$-\mathrm{ESBL}^{-E^{25}}(\%)$ & $21(14)$ & 7 (19) & $10(12)$ & $4(14)$ \\
\hline -Others (\%) & 32 (19) & $12(25)$ & $13(18)$ & 7 (23) \\
\hline Prior $\mathrm{ABX}^{26}, \mathrm{n}(\%)$ & $82(72)$ & $18(72)^{27}$ & $42(67)^{28}$ & $22(88)^{29}$ \\
\hline Concomitant ABX, n (\%) & 35 (31) & $9(36)^{30}$ & $20(32)^{31}$ & $6(24)^{32}$ \\
\hline Tigecycline treatment, mean days & 14 & $15^{33}$ & $11^{34}$ & $21^{35}$ \\
\hline
\end{tabular}

${ }^{1}$ Skin and skin structure infections; ${ }^{2}$ Intra-abdominal infections; ${ }^{3}$ Ventilator-associated pneumonia; ${ }^{4 \text { vs. } 5 \text { vs. } 6 \text { No significant difference was found; }}$

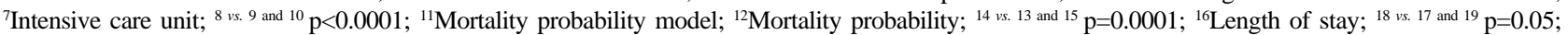
${ }^{20}$ Multidrug-resistant Acinetobacter spp.; ${ }^{22}$ vs. 21 and ${ }^{23} \mathrm{p}=0.0005$; ${ }^{24}$ Methicillin-resistant S.aureus; ${ }^{25}$ Extended-spectrum $\beta$-lactamases-producing enterobacteriaceae; ${ }^{26}$ Antibiotic; ${ }^{27}$ vs. $28, v s .29$ No significant difference was found; ${ }^{30}$ vs. 31 ,vs. 32 No significant difference was found; ${ }^{35}$ vs. 33 and 34 p $=0.015$.

Table 2. Proportion of patients with clinical success (CS) criteria.

\begin{tabular}{|c|c|c|c|c|}
\hline \multirow[t]{2}{*}{ Characteristics } & \multirow[t]{2}{*}{ Global } & \multicolumn{3}{|c|}{ Indications } \\
\hline & & Type I & TypeII & TypeIII \\
\hline All patients, CS/total (\%) & $86 / 113(76)$ & $22 / 25(88)^{1}$ & $42 / 63(66)^{2}$ & $21 / 25(84)^{3}$ \\
\hline \multicolumn{5}{|l|}{ Prior ABX ${ }^{4}, \mathrm{CS} /$ total (\%) } \\
\hline -Yes & $61 / 82(74)^{5}$ & $16 / 18 \quad(89)$ & $27 / 42(64)$ & $18 / 22 \quad(82)$ \\
\hline$-\mathrm{No}$ & $24 / 31(74)^{6}$ & $6 / 7 \quad(87)$ & $14 / 21$ & $2 / 3 \quad(66)$ \\
\hline \multicolumn{5}{|l|}{ Concomitant ABX, CS/total (\%) } \\
\hline -Yes & $20 / 35(57)^{7}$ & 8/9 (89) & $10 / 20(50)$ & $4 / 6 \quad(66)$ \\
\hline$-\mathrm{No}$ & $74 / 78(95)^{8}$ & $14 / 16 \quad(87)$ & $35 / 43$ (82) & 19/19 (100) \\
\hline \multicolumn{5}{|l|}{ Isolates, CS/total (\%) } \\
\hline$-A B-M D R{ }^{9}$ & $50(67)^{12}$ & $9(75)$ & 32 (64) & 9 (75) \\
\hline- MRSA $^{10}$ & $17(68)^{13}$ & 7 (77) & $6(66)$ & $4 \quad(57)$ \\
\hline$-\mathrm{ESBL}_{-} \mathrm{EB}^{11}$ & $17(81)^{14}$ & $7(100)$ & 7 (70) & $3(75)$ \\
\hline -Others & $23(79)$ & $9(100)$ & $8(61)$ & $6(86)$ \\
\hline Tigecycline treatment, mean days & 16 & $15^{15^{2}}$ & $12^{16^{(2)}}$ & $22^{17}$ \\
\hline
\end{tabular}

1 and 3 vs. ${ }^{2} \mathrm{p}=0.032$; ${ }^{4}$ Antibiotic; ${ }^{5 \text { vs. }}{ }^{6}$ No significant difference was found; ${ }^{7 \text { vs. } 8} \mathrm{p}=<0.00001$; ${ }^{9}$ Multidrug-resistant Acinetobacter spp.; ${ }^{10}$ Methicillin-resistant S.aureus; ${ }^{11}$ Extended-spectrum $\beta$-lactamases-producing enterobacteriaceae; ${ }^{12 \text { vs. } 13 \text { and } 14}$ No significant difference was found; ${ }^{17 \text { vs. } 15 \text { and } 16} \mathrm{p}=0.015$.

The high intrapulmonary concentration of tigecycline [5] as well as the prevalence in our study of VAP due to MDRAcinetobacter spp. (61\%), ESBL-producing EB (12\%), and MRSA (11\%) -all of them susceptible to tigecycline (at least in vitro)- seem to explain the reasons why physicians choose the drug to treat these patients. Furthermore, while VAP due to MRSA or ESBL-producing EB have other therapeutic options (linezolid [6] and carbapenems [7], respectively), VAP due to Acinetobacter spp. resistant to almost all groups of commercially available antimicrobials, creates a serious clinical problem [8]. Several studies, most of them with small samples, have shown good outcomes in patients with VAP due to MDRAcinetobacter spp. treated with colistin [9], high-dose ampicillin-sulbactam [10], tetracyclines [11](doxycycline or minocycline) or aerosolized tobramycin [12]. However, the recorded clinical experience with these antibiotics is still limited 
and the reported studies of their use in the treatment of VAP are not randomized controlled clinical trials. Tigecycline can thus be considered an alternative.

The proportion of global CS in our patients was $76 \%$. Tigecycline's CS proportion observed in patients with type I indications (88\%) was similar to those reported in the phase III clinical trials (86.5\% in cSSSI and $86.1 \%$ in cIAI) [2].

The administration of prior antibiotic therapy has not been associated with significant differences in the proportion of CS. In contrast, patients who received concomitant antibiotic treatment showed a CS rate significantly lower than patients who did not received concomitant antibiotic treatment (57\% vs. 95\%, respectively, $\mathrm{p}=0.05$ ). These findings cannot be easily explained but the small sample size might be the reason behind this finding, and does not allow us to draw any definite conclusion.

The CS rate for patients with VAP was significantly less than the global rate -66\%- (MDR-Acinetobacter spp. 64\%, MRSA 66\% and ESBL-producing EB 70\%), reflecting a severity of illness significantly higher than that of the other indications. Ninety-eight percent (98\%) of patients with VAP were in the ICU, as expected, and they had a mortality rate of 38\%, less than that predicted by the MPM II $(55 \%)$. A possible explanation is that the MPM $\mathrm{II}_{0}$ overestimates the mortality of patients who have probabilities of death of $\geq 40 \%$ [13]. In that sense, Heyland et al. [14] reported that in patients with VAP there is a $20 \%$ to $55 \%$ mortality rate which increases to $76 \%$ if the infection is caused by MDR pathogens, evidencing the fact that our data should be taken into account.

Adverse event and microbiological eradication data, was not specifically collected in our study.

In summary, "off label” use of tigecycline in Argentina is frequent especially in severe infections, such as VAP due to MDR-Acinetobacter spp., based on the pharmacological and microbiological profile of the drug.

Finally, we know that our study is not a rigorous trial with specific inclusion and exclusion criteria, close case monitoring and strict follow-up, however, the results obtained in this prospective observational study provide some initial evidence that tigecycline may be an acceptable option for indications that have not yet been approved.

\section{Tigecycline Initial Use Registry Group}

Members of the Tigecycline Initial Use Registry Group include Liliana Calanni. (Hospital Dr. Eduardo Castro Rendón, Neuquén, Argentina); Francisco Nacinovich (Instituto Cardiovascular de Buenos Aires e Instituto Medico de Alta Complejidad, Buenos Aires, Argentina); Martin Christin and Claudia Tosello (Instituto Medico de Alta Complejidad, Buenos Aires, Argentina); Marcelo del Castillo and Andrea Mora (FLENI Instituto de Investigaciones Dr.Raúl Carrea,
Buenos Aires, Argentina); Jorge Calderón (Policlínico Neuquén, Neuquén, Argentina); Jorge Vergara (Hospital Ramos Mejía, Buenos Aires, Argentina); Walter Vázquez (Hospital Español, Mendoza, Argentina); Ana Colombini (Clínica San Camilo, Buenos Aires, Argentina); Pablo Bonvehí (CEMIC, Buenos Aires, Argentina); Paulo Chinchilla (Clínica Ciudad, Buenos Aires, Argentina); Laura Barcán (Hospital Italiano, Buenos Aires), Daniel Curcio and Francisco Fernández (Sanatorio San José, Buenos Aires, Argentina).

\section{References}

1. Quinteros M., Radice M., Famiglietti A., et al. Análisis de la sensibilidad a los antimicrobianos en los aislamientos de pacientes internados años 2004-2005. Boletín de la Asociación Argentina de Microbiología 2006;172:13-6.

2. Pankey G.A. Tigecycline. J Antimicrob Chemother. 2005;56(3):470-80

3. Lemeshow S., Teres D., Klar J., et al. Mortality probability models (MPM II) based on an international cohort of intensive care patients. JAMA 1993;270:2478-86.

4. Pritchard R.H. Off-Label Uses of Approved Drugs: A New Compromise Is Needed, (March 25, 1999) available at leda.law.harvard.edu/leda.

5. Conte J.E., Golden J.A., Kelly M.G., Zurlinden E. Steady-state serum and intrapulmonary pharmacokinetics and pharmacodynamics of tigecycline. Int $\mathrm{J}$ Antimicrob Agents 2005;25(6):523-9.

6. Grau S., Alvarez-Lerma F., del Castillo A., et al. Cost-effectiveness analysis of the treatment of ventilator-associated pneumonia with linezolid or vancomycin in Spain. J Chemother 2005;17(2):203-11.

7. Lorente L., Lorenzo L., Martin M., et al. Meropenem by continuous versus intermittent infusion in ventilator-associated pneumonia due to Gram-negative bacilli. Ann Pharmacother. 2006;40(2):219-23.

8. Li J., Nation R.L., Turnidge J.D., et al. Colistin: the re-emerging antibiotic for multidrug-resistant Gram-negative bacterial infections. Lancet Infect Dis 2006;6(9):589-601.

9. Kallel H., Hergafi L., Bahloul M., et al. Safety and efficacy of colistin compared with imipenem in the treatmentof ventilatorassociated pneumonia: a matched case-control study. Intensive Care Med 2007;33(7):1162-7.

10. Betrosian A.P., Frantzeskaki F., Xanthaki A., Georgiadis G. Highdose ampicillin-sulbactam as an alternative treatment of lateonset VAP from multidrug-resistant Acinetobacter baumannii. Scand J Infect Dis 2007;39(1):38-43.

11. Wood G.C., Hanes S.D., Boucher B.A., et al. Tetracyclines for treating multidrug-resistant Acinetobacter baumannii ventilator-associated pneumonia. Intensive Care Med 2003;29(11):2072-6.

12. Hallal A., Cohn S.M., Namias N., et al. Aerosolized tobramycin in the treatment of ventilator-associated pneumonia: a pilot study. Surg Infect (Larchmt) 2007;8(1):73-82.

13. Rué M., Artigas A., Alvarez M., et al. Performance of the mortality probability models in assesing severity of iones during the first week in the intensive care unit. Crit Care Med 2000;28(8):2819-24.

14. Heyland D.K., Cook D.J., Griffith L., et al. The attributable morbidity and mortality of ventilator-associated pneumonia in the critically ill patient. The Canadian Critical Trials Group. Am J Respir Crit Care Med 1999;159(4 Pt 1):1249-56. 